Article

\title{
Sol-Gel-Processed Organic-Inorganic Hybrid for Flexible Conductive Substrates Based on Gravure-Printed Silver Nanowires and Graphene
}

\author{
Xinlin $\mathrm{Li}^{1,+}{ }^{+}$, Nahae Kim ${ }^{2,+}$, Seongwook Youn ${ }^{3}$, Tae Kyu An ${ }^{4, * \mathbb{C}}$, Juyoung Kim ${ }^{2, *}$, \\ Sooman Lim ${ }^{5, *(D)}$ and Se Hyun Kim 1,6,* \\ 1 Department of Mechanical Engineering Science, Yeungnam University, Gyeongsan, Gyeongbuk 38541, \\ Korea; xinlin0618@gmail.com \\ 2 Department of Advanced Materials Engineering, Kangwon National University, Samcheok 25931, Korea; \\ kjr2856@naver.com \\ 3 Department of Software, Korea National University of Transportation, 50 Daehak-Ro, Chungju 27469, Korea; \\ youn@ut.ac.kr \\ 4 Department of Polymer Science and Engineering \& IT Convergence, Korea National University of \\ Transportation, 50 Daehak-Ro, Chungju 27469, Korea \\ 5 Graduate School of Flexible and Printable Electronics, Chonbuk National University, Jeonju 54896, Korea \\ 6 School of Chemical Engineering, Yeungnam University, Gyeongsan, Gyeongbuk 38541, Korea \\ * Correspondence: taekyu1985@ut.ac.kr (T.K.A.); juyoungk@kangwon.ac.kr (J.K.); smlim@jbnu.ac.kr (S.L.); \\ shkim97@yu.ac.kr (S.H.K.) \\ + Equally contributed as first authors.
}

Received: 24 December 2018; Accepted: 16 January 2019; Published: 17 January 2019

check for updates

\begin{abstract}
In this study, an organic-inorganic (O-I) nanohybrid obtained by incorporating an alkoxysilane-functionalized amphiphilic polymer precursor into a $\mathrm{SiO}_{2}-\mathrm{TiO}_{2}$ hybrid network was successfully utilized as a buffer layer to fabricate a flexible, transparent, and stable conductive substrate for solution-processed silver nanowires (AgNWs) and graphene under ambient conditions. The resulting O-I nanohybrid sol (denoted as AGPTi) provided a transmittance of the spin-coated AgNWs on an AGPTi-coated glass of $99.4 \%$ and high adhesion strength after a $3 \mathrm{M}$ tape test, with no visible changes in the AgNWs. In addition, AGPTi acted as a highly functional buffer layer, absorbing the applied pressure between the conductive materials, AgNWs and graphene, and rigid substrate, leading to a significant reduction in sheet resistance. Furthermore, gravure-printed AgNWs and graphene on the AGPTi-based flexible substrate had uniform line widths of $490 \pm 15$ and $470 \pm 12 \mu \mathrm{m}$, with 1000-cycle bending durabilities, respectively.
\end{abstract}

Keywords: conductive substrate; gravure printing; flexible electronics; and nanomaterials

\section{Introduction}

Recently, flexible electronic devices, such as displays, energy harvesting systems, memories, sensors, and many more [1-7], have attracted significant interest. Intensive studies have led to the development of prototype flexible devices, enabled by the fabrication of novel materials, development of processing techniques, and simulations on the device physics. However, several scientific and technical challenges should be overcome to commercialize the devices [8,9]. In particular, future flexible electronics will require solution-based printing processes instead of vacuum and photolithographic processes, which have been widely used in the fabrication of the current electronic devices [10-15]. The printing processes enable direct patterning of inks on various substrates (e.g., glass, polymer, metal foil, and paper) without auxiliary deposition and removal of a photoresist, which provides valuable 
advantages including simplicity, low cost, and large-area solution processability. Therefore, significant progress is required in the development of printable materials for flexible electronic devices [16-20].

As electronic devices are fabricated on substrates, flexible conductive substrates are the basis for the successful fabrication of flexible electronics. Among the flexible conductive substrates, indium tin oxide (ITO)-coated polymers (such as polyethylene terephthalate (PET), polyethylene naphthalate (PEN), and poly(ethylene succinate) (PES)) have been widely used owing to the high transparency and conductivity of the ITO layer [21-25]. However, there are disadvantages, as ITO is rare, brittle, and requires vacuum processing, which can hinder its applicability to the rollable electronics. Therefore, alternative conductive materials, such as carbon nanomaterials, metal nanoparticles and nanowires, and organic conducting polymers, are extensively investigated through solution processes [26-30].

Prior to the deposition of conductive materials on a polymer substrate, the introduction of a buffer layer is essential to improve surface characteristics of the polymer substrate to fabricate a smooth surface, and to fill in pinholes and to enhance the adhesion between the conductive materials and substrate [31-33]. In addition, the buffer layer should improve the mechanical, thermal, and chemical stabilities of the underlying substrate [34-36]. Various polymers can be easily formed as buffer layers through roll-to-roll and printing processes. However, their resistance to mechanical impacts (e.g., abrasion) and excessive thermal and chemical treatments still needs to be improved. An inorganic coating fabricated by gas-phase vacuum deposition (chemical vapor deposition (CVD), physical vapor deposition (PVD)) provides excellent abrasion strength as well as thermal and chemical resistance. However, several factors, such as the high cost, weak adhesion, and brittleness, hinder its application in flexible electronics [37].

O-I nanohybrid materials were utilized as they exhibit desirable characteristics of both inorganic (thermal stability, mechanical integrity, etc.) and organic (flexibility, simple processing, specific chemical functionality, etc.) materials [38-41]. In particular, the sol-gel-derived O-I nanohybrids enabled simple processing. Depending on the choice of precursors and their combinations, it is possible to obtain nanohybrid films with variable physicochemical properties. Therefore, the sol-gel deposition of thin films has been applied to various fields owing to the advantages such as abrasion resistance, anticorrosion, antireflection, and self-cleaning [42]. These advantages are attributed to the stable covalent bonds formed at the interface between the O-I nanohybrid and substrate with surface hydroxyl groups. The stable covalent bonds originate from the sol-gel reaction route. Common sol-gel reaction routes during the sol-gel process are:

$$
\begin{gathered}
\mathrm{Si}-(\mathrm{OR})_{n}+\mathrm{H}_{2} \mathrm{O} \leftrightarrow \mathrm{Si}-\mathrm{OH}+\mathrm{ROH} \text { : hydrolysis, } \\
\mathrm{Si}-\mathrm{OH}+\mathrm{Si}-\mathrm{OH} \leftrightarrow \mathrm{Si}-\mathrm{O}-\mathrm{Si}+\mathrm{H}_{2} \mathrm{O} \text { : water condensation, } \\
\mathrm{Si}-\mathrm{OH}+\mathrm{Si}-\mathrm{OR} \leftrightarrow \mathrm{Si}-\mathrm{O}-\mathrm{Si}+\mathrm{ROH} \text { : alcohol condensation. }
\end{gathered}
$$

Substitution of the hydroxyl groups $(-\mathrm{OH})$ for alkoxide groups $(-\mathrm{OR})$ of the silane compound occurs during the hydrolysis, owing to the interaction of alkoxide groups with $\mathrm{H}_{2} \mathrm{O}$; subsequently, water condensation or/and alcohol condensation occur, producing $\mathrm{Si}-\mathrm{O}-\mathrm{Si}$ covalent bonds, water, and alcohol, respectively. The condensations occur not only in the O-I nanohybrid bulk but also at the interface with the substrate. Although alkoxysilane-based $\mathrm{O}-\mathrm{I}$ nanohybrids are advantageous in several applications, some issues need to be overcome to enable commercialization. For example, O-I hybrid sols are prepared using only alkoxysilane compounds, often suffering from aggregation, which implies that the gelation reaction unexpectedly occurs during the film processing. In order to address the gelation issue, amphiphilic molecules have been used as a dispersion agent to obtain stable sols. However, amphiphilic materials, such as the commonly used surfactants, do not participate in the reaction. Therefore, they should be removed after the preparation to avoid deterioration in the mechanical properties of the O-I hybrid materials [43]. In this regard, it is required to synthesize amphiphilic materials that can be chemically bonded to alkoxysilanes, yielding stable and mechanically strong O-I hybrid materials. The alkoxysilane-functionalized amphiphilic polymer (AFAP) polymer 
used in this study consists of polyethylene oxide-based hydrophilic and polypropylene oxide-based hydrophobic segments with reactive alkoxysilane groups at both ends. It could be expected that the amphiphilic polymer backbones of AFAP colloidally stabilize O-I nanohybrid sols formed during the hydrolytic condensation polymerization without a dispersion agent. Moreover, their alkoxysilane groups can chemically react with organosilanes, thereby leading to $\mathrm{Si}-\mathrm{O}-\mathrm{Si}$ networks, which can improve the miscibility between the organic and inorganic components in the resulting O-I nanohybrid materials. Simultaneously, the amphiphilic polymer backbone of the alkoxysilane precursor facilitates the formation of a flexible homogeneous film after the curing process.

In this study, in order to fabricate a flexible conductive substrate based on solution-processed silver nanowires (AgNWs) and graphene, we demonstrate an organic-inorganic (O-I) nanohybrid by incorporating an alkoxysilane-functionalized amphiphilic polymer (AFAP) precursor into a $\mathrm{SiO}_{2}-\mathrm{TiO}_{2}$ hybrid network. The resulting O-I nanohybrid sol can be deposited to a uniform film on substrates, such as glass and PET, by spin- and bar-coating methods. Subsequently, annealing $\left(105{ }^{\circ} \mathrm{C}\right.$ for $30 \mathrm{~min}$ ) hardens the film to achieve chemical and mechanical strength and transparency. AgNWs and graphene conductive materials are formed on the O-I nanohybrid-treated substrates by spray-coating and gravure printing, which enable fabrication of the micropatterns of the materials. The O-I nanohybrid-coated flexible conductive substrates exhibit high conductivities and good adhesion properties in both cases of AgNWs and graphene, with excellent bending stabilities at a radius of curvature of $5 \mathrm{~mm}$.

\section{Experimental Methods}

\subsection{Synthesis of the O-I Nanohybrid}

In the synthesis of AFAP precursors [38], isophorone diisocyanate (IPDI, Aldrich Chemical Co., St. Louis, MS, USA), glycerol (Gc, $M_{\mathrm{w}}: 92.09 \mathrm{~g} / \mathrm{mol}$, Aldrich Chemical Co., St. Louis, MS, USA), (3-aminopropyl)triethoxysilane (APTES, Aldrich Chemical Co.), and poly(ethylene glycol) (PEG, $M_{\mathrm{W}}=300 \mathrm{~g} / \mathrm{mol}$, Aldrich Chemical Co., St. Louis, MS, USA) were used as received, without further purification. Dibutyltin dilaurate (DBTDL, Aldrich Chemical Co., St. Louis, MS, USA) was used as a catalyst in the precursor synthesis. (3-Glycidyloxypropyl)trimethoxysilane (GPTMS, 9\%, Aldrich Chemical Co., St. Louis, MS, USA), propyltrimethoxysilane (PTMS, 97\%, Aldrich Chemical Co., St. Louis, MS, USA), titanium(IV) isopropoxide (TTiP, 97\%, Aldrich Chemical Co., St. Louis, MS, USA), ethanol (Aldrich Chemical Co., St. Louis, MS, USA), and hydrochloric acid ( $\mathrm{HCl}$, ACS reagent, 37\%, Aldrich Chemical Co., St. Louis, MS, USA) were used, as received, for the preparation of O-I hybrid sols. Gc and PEG were dried under a reduced pressure for $24 \mathrm{~h}$ at $80{ }^{\circ} \mathrm{C}$ before use. The specific synthesis of the $\mathrm{O}-\mathrm{I}$ nanohybrid colloidal solution is presented in a previous report [39]. In order to prepare the O-I nanohybrid sols, the AFAP precursors were mixed with GPTMS, PTMS, TTiP, ethanol, and water $(0.1 \mathrm{M} \mathrm{HCl}(\mathrm{aq}))$, at a mass ratio of 1:1:1:4:0.4, and stirred at $60^{\circ} \mathrm{C}$ for $48 \mathrm{~h}$ to induce the hydrolytic hydrolysis-condensation reaction. The resultant O-I hybrid sol is referred to as AGPTi, whereas the O-I hybrid sol without AFAP is denoted as GPTi. During the reaction, no macrophase separation and precipitation occurred in the solution without a chelating agent or stabilizer.

\subsection{Fabrication of Conductive Films}

AgNWs and water-dispersed graphene were used as conductive materials in this study. The AgNWs were synthesized using ethylene glycol (EG) in the presence of $\mathrm{ZnCl}_{2}, \mathrm{Fe}\left(\mathrm{NO}_{3}\right)_{3} \cdot \mathrm{H}_{2} \mathrm{O}$, and poly( $N$-vinylpyrrolidone), as reported in [40]. The resulting AgNWs had diameters of 40-80 nm and lengths of 30-80 $\mu \mathrm{m}$. The prepared AgNWs (0.06 wt \%) were dispersed in isopropyl alcohol (IPA) for coating onto glass and O-I nanohybrid films. A water-dispersible graphene paste ink was purchased from MExplorer (Ansan, Korea), in which graphene flakes are homogenously dispersed in deionized (DI) water without any organic binders. Bare glass $\left(3.5 \times 3.5 \mathrm{~cm}^{2}\right)$ substrates were cleaned in boiled acetone and subsequently sonicated several times in acetone/isopropyl alcohol/distilled water. 
The cleaned bare glass was exposed to a UV ozone cleaner for 5 min for further hydrophilization. In order to fabricate transparent conductive thin films, dispersions of $50 \mathrm{~mL}$ of AgNWs (AgNWs/bare glass) and $100 \mathrm{~mL}$ of graphene mixed with IPA (volume ratio: 1:1) (graphene/bare glass) were spray-coated on bare glasses, used as references. For functionalization of the conductive thin film, both AGPTi and GPTi O-I nanohybrid sols mixed with propylene glycol monomethyl ether acetate (PGMEA, volume ratio: 1:1) were spun on a clean bare glass at $9000 \mathrm{rpm}$ for $3 \mathrm{~min}$ at room temperature, followed by annealing at $105{ }^{\circ} \mathrm{C}$ for $30 \mathrm{~min}$, as shown in Figure 1. On the O-I nanohybrid films on the bare glasses, the AgNW (AgNWs/O-I nanohybrid/glass) and graphene (graphene/O-I nanohybrid/glass) dispersions were spray-coated under the same conditions used for the references (Figure 1a). The conductive films were then mechanically pressed $(\mathrm{P})$ at $10 \mathrm{MPa}$ for $10 \mathrm{~min}$ to increase their conductivities. Finally, electrodes with low surface roughnesses, high transparencies, and low resistances were fabricated, with AgNWs (P-AgNWs/O-I nanohybrid/glass) and graphene (P-graphene/O-I nanohybrid/glass), as shown in Figure 1b. In order to apply these materials to gravure printing $(G)$, the purchased AgNWs and graphene ink mixed with IPA (60 wt \%) were employed. Optimization of printing parameters, such as ink viscosity, surface energy, and printing speed, enabled printing of conductive lines of AgNWs and graphene on both AGPTi- and GPTi O-I nanohybrid-coated PET substrates (G-AgNWs/O-I nanohybrids/PET). In this process, a copper gravure pattern manufactured by photolithography and chemical etching was utilized as a gravure pattern in trenches.

(a)

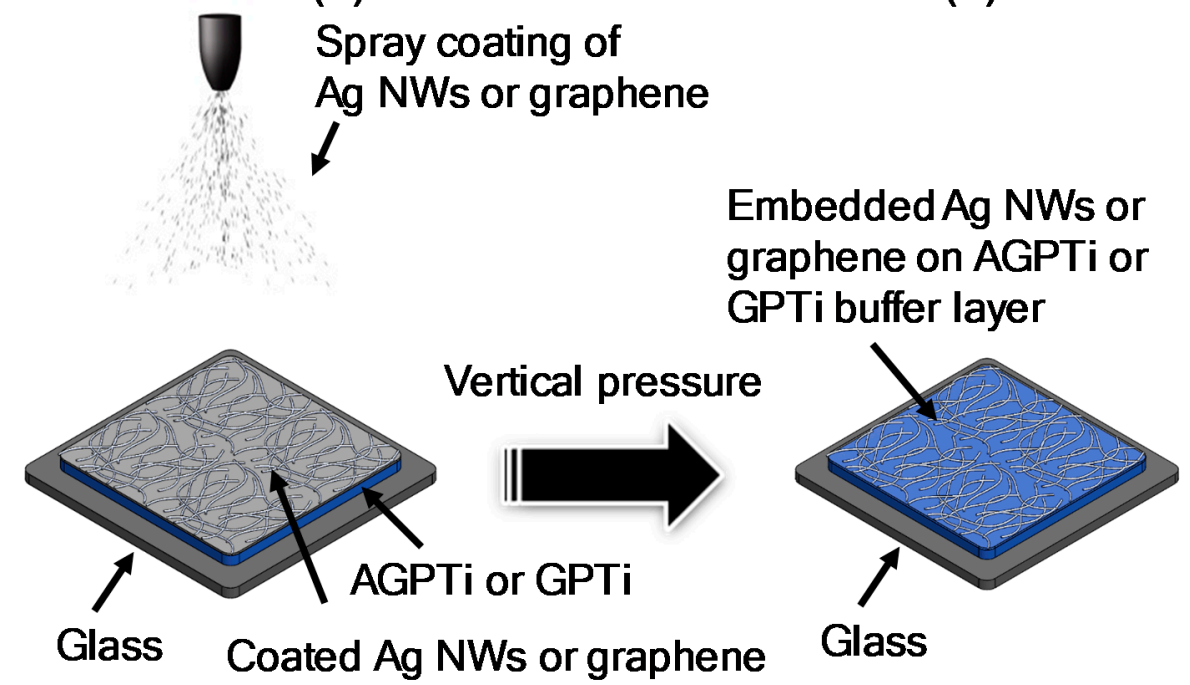

Figure 1. Fabrication of AgNW (or graphene) electrodes with high conductivities and adhesion strengths by mechanical pressing techniques on the AGPTi (or GPTi) buffer layer. (a) spray coating of AgNWs or graphene, (b) applying pressure to the film.

\subsection{Characterization of the Conductive Films}

In order to characterize their optical and electrical properties, the transmittances of the AgNW- and graphene-based conductive films were measured using a spectrophotometer (GENESYS 10S UV-vis, Thermo Fisher Scientific ${ }^{\mathrm{TM}}$ (Seoul, Korea), showing spectral bandwidth of $1.8 \mathrm{~nm}$ and wavelength accuracy of $\pm 1.0 \mathrm{~nm}$ ), in which samples were measured in a range of wavelengths from 190 to $1100 \mathrm{~nm}$. The sheet resistances (Rs) were measured using the four-probe technique (Loresta EP MCP-T360, Mitsubishi chemical, Tokyo, Japan, determination range: $10^{-2}-10^{6} \Omega$ ). Optical microscopy (OM, Nikon ECLIPSE LV100ND, Nikon, Tokyo, Japan, Max. sample size: $150 \times 150 \mathrm{~mm}^{2}$ ). Atomic force microscopy (AFM, Veeco DI Dimension 3100 with Nanoscope V, Al-coated Si tips which were purchased from Nanosensors ${ }^{\mathrm{TM}}$, Neuchâtel, Switzerland) was used to measure surface properties. Tapping-mode was used to take AFM images with Al-coated Si tips which were purchased from NanosensorsTM 
(Neuchâtel, Switzerland, thickness: $4.0 \pm 1 \mu \mathrm{m}$; length: $125 \pm 10 \mu \mathrm{m}$; width: $30 \pm 7.5 \mu \mathrm{m}$; resonance frequency: 204-497 kHz; force constant: 10-130 N/m; tip height: 10-15 $\mu \mathrm{m}$ ). Scanning electron microscopy (SEM, Hitachi S4800, Hitachi, Tokyo, Japan, resolution: $1.0 \mathrm{~nm}$ at $15 \mathrm{kV}, 1.4 \mathrm{~nm}$ at $1 \mathrm{kV}$, deacceleration mode) were used to measure the surface roughness and topography. In order to determine the mechanical properties, a taping test with a $3 \mathrm{M}$ tape was applied to the coated films, which were pressed evenly and simultaneously detached.

\section{Results and Discussion}

In order to investigate the effect of AFAP on the physical and chemical properties of the $\mathrm{SiO}_{2}-\mathrm{TiO}_{2}$-based $\mathrm{O}-\mathrm{I}$ nanohybrid for the conductive substrate, AGPTi (with AFAP) and GPTi (without AFAP) O-I nanohybrid sols were coated onto glass and PET substrates through spin-casting and gravure printing. First, we characterized the transmittances of the glass substrates coated with the AGPTi and GPTi O-I nanohybrids, as a high transparency is very desirable in optoelectronic devices. Figure 2 shows the transmittances of the spin-coated AGPTi/glass (99.4\% at $550 \mathrm{~nm}$ ) and GPTi/glass $(99.2 \%$ at $550 \mathrm{~nm})$, which are comparable to that of the bare glass. In addition, both AGPTi/glass and GPTi/glass did not become hazy or degrade upon light irradiation. In general, nanostructured thin films can be efficiently deposited by spray-coating, providing a cheap large-area deposition of AgNWs and graphene $[44,45]$. Therefore, the fabrications of conductive thin films of AgNWs and graphene were performed on the samples by simple spray-coating processes under ambient conditions, as shown in Figure 1.

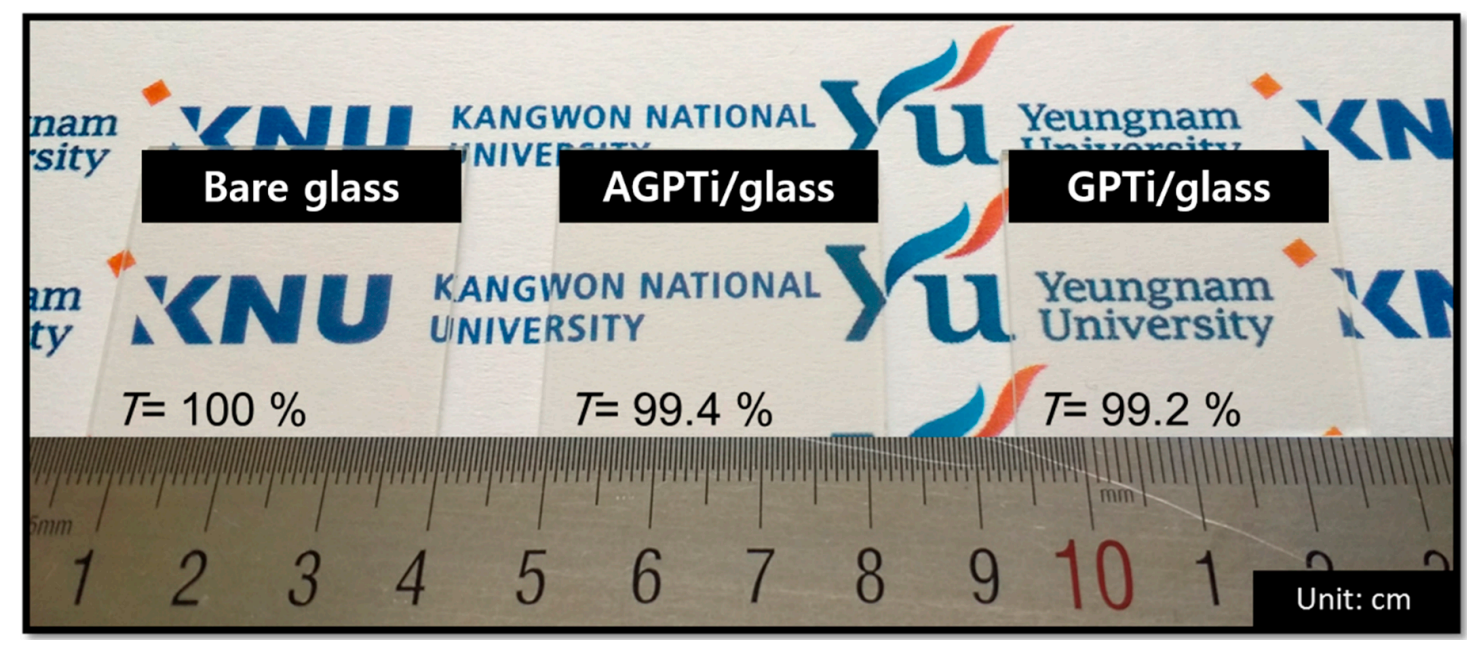

Figure 2. Images of the bare glass and AGPTi- and GPTi-coated glasses used for the fabrication of highly transparent electrodes.

In order to investigate their adhesive strengths, 3M taping tests were performed for the thin films on the bare glass and AGPTi- and GPTi-coated glass substrates. The results showed low adhesions of AgNWs/glass (Figure 3a,a-1) and graphene/glass (Figure 3b,b-1). This tendency is generally observed for noble metals with weak adhesions on glass [46,47]. By contrast, the substrates functionalized by the deposited AGPTi and GPTi O-I nanohybrid layers had higher adhesion strengths; no visible changes in the AgNWs (Figure 3c,c-1 for AGPTi and Figure 3e,e-1 for GPTi) and graphene (Figure 3d,d-1 for AGPTi and Figure 3f,f-1 for GPTi) were observed after the taping test. The AGPTi and GPTi O-I nanohybrids had an important role as buffer matrices against the applied pressure. The AgNWs and graphene penetrated these polymer layers, leading to an enhancement in adhesion and, thus, to maintenance of sheet resistance after the taping test. Moreover, the pressed AgNWs and graphene on AGPTi and GPTi led to reductions in their sheet resistances, from 5 to $1.5 \Omega$ /sq and from 700 to $600 \Omega / \mathrm{sq}$, respectively (Figure 4). This is mainly attributed to the enhanced connection of the wire-to-wire junctions of the AgNWs during the pressing process [48]. In the case of graphene, the application of 
pressure enlarges the contact area between graphene particles, leading to a significant reduction in the additional resistance attributed to individual particles [49]. These results are demonstrated by optical microscopy (OM) and scanning electron microscopy (SEM) (insets) images of the AgNWs and graphene before (Figure $5 a, c$ ) and after (Figure $5 b, d$ ) the pressing process. A similar tendency was observed for the GPTi O-I nanohybrid-coated film, which is not shown in this figure.

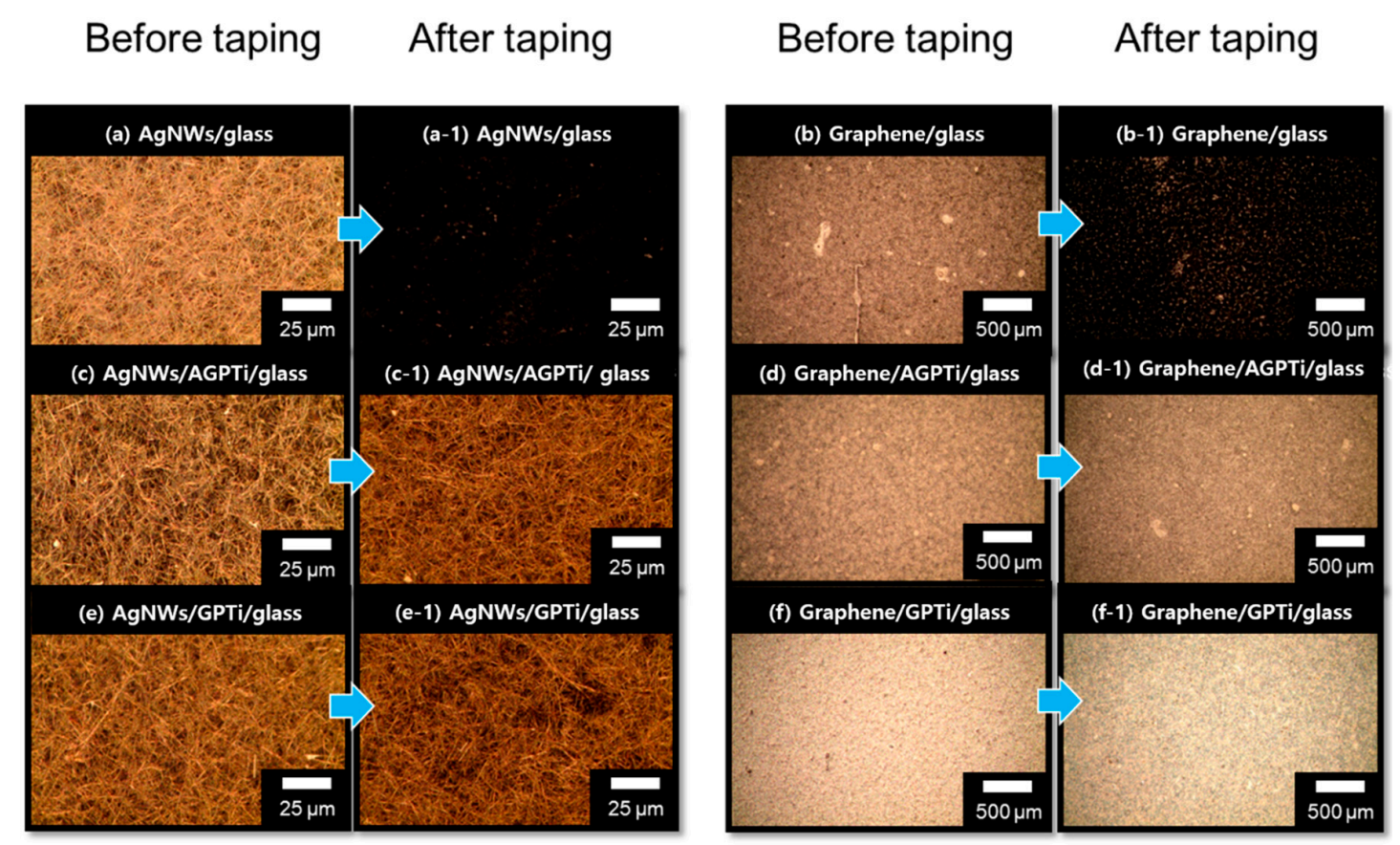

Figure 3. Optical microscopic images before and after the 3M taping tests on AgNWs/bare glass (a,a-1), graphene/bare glass (b,b-1), AgNWs/AGPTi (c,c-1), graphene/AGPTi (d,d-1), AgNWs/GPTi (e,e-1), and graphene/GPTi (f,f-1).

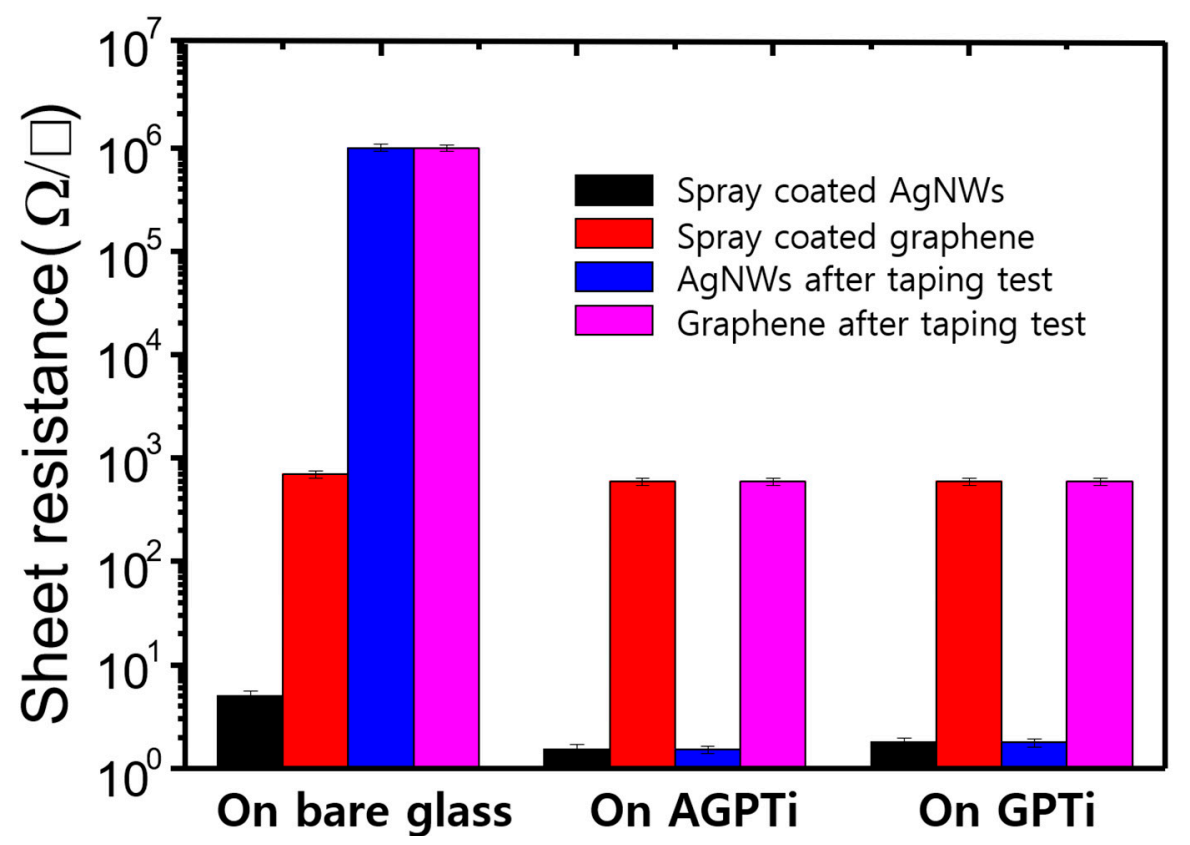

Figure 4. Changes in the sheet resistances of the AgNWs and graphene after the taping tests on the bare glass and AGPTi- and GPTi-coated glasses. 

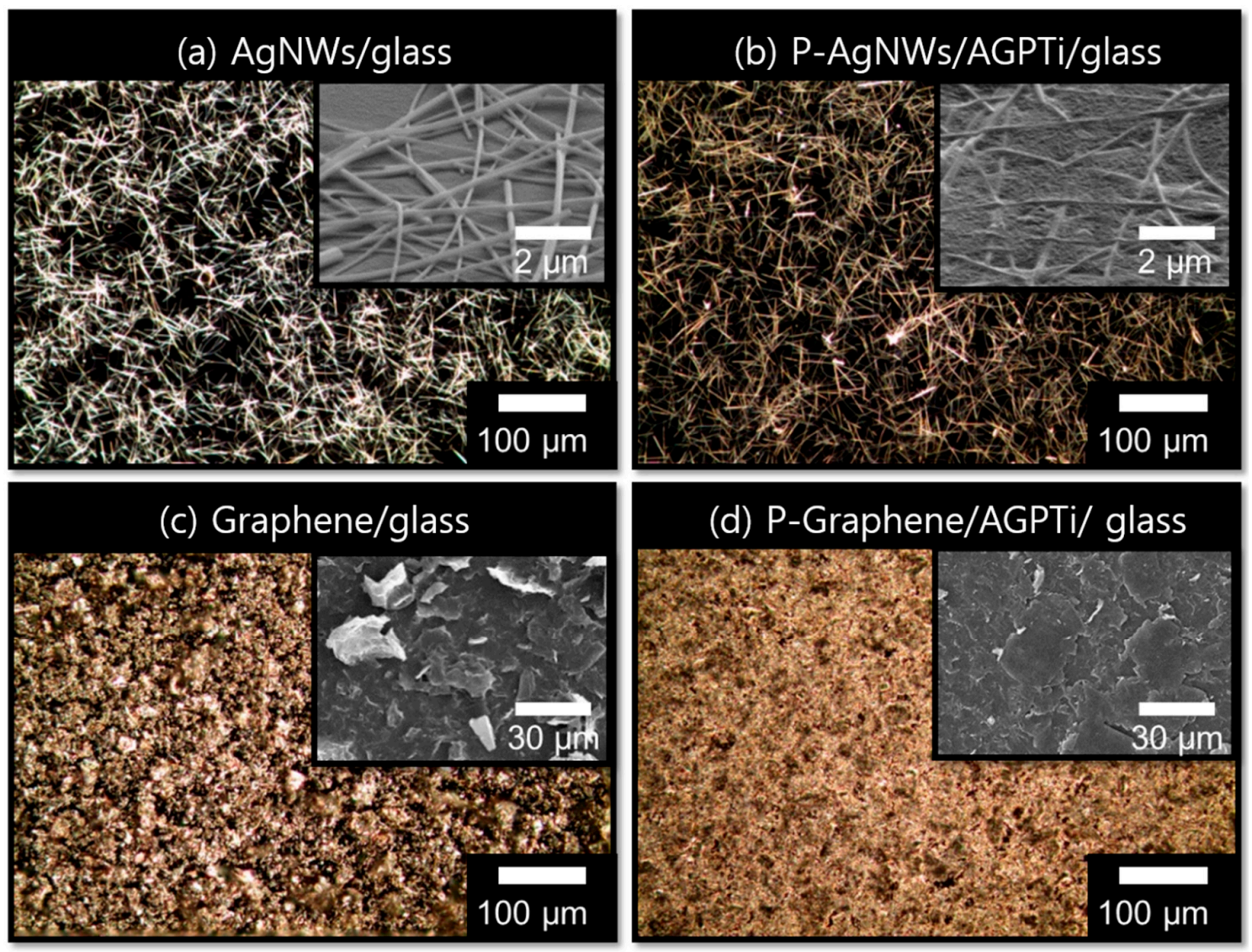

Figure 5. OM and SEM images of the AgNWs and graphene before $(\mathbf{a}, \mathbf{b})$ and after $(\mathbf{c}, \mathbf{d})$ the mechanical pressing, respectively.

An AFM analysis (Figure 6) demonstrated that the pressing processes on the AGPTi and GPTi O-I nanohybrid films contributed to the decreases in the surface roughnesses of the AgNWs and graphene. In general, AgNWs exhibit a high surface roughness due to their intrinsic wire structure. The root-mean-square $\left(R_{\mathrm{q}}\right)$ roughness of AgNWs/glass was $15 \mathrm{~nm}$, corresponding to the diameters of the wires $(60-100 \mathrm{~nm}) . \quad R_{\mathrm{q}}$ significantly decreased to $2.5 \mathrm{~nm}$ after the pressing processes on the AGPTi (Figure 6a,a-1) and GPTi (Figure 6b,b-1) O-I nanohybrids, without sticking-out and loss of transparency. In a previous study, embedment of AgNWs on a predeposited film (e.g., oly(3,4-ethylenedioxythiophene):poly(styrenesulfonate) (PEDOT:PSS)) led to a decrease in surface roughness [50]. However, this affected the transparency owing to the trade-off relationship considering the original color of the conductive polymer [50]. In this study, this issue was overcome using the highly transparent films of the AGPTi and GPTi O-I nanohybrids, enabling the decreases in the surface roughnesses without loss of transparency. A similar trend was observed for the case of graphene. The roughness of graphene on the AGPTi (Figure $6 c, c-1$ ) and GPTi (Figure 6d,d-1) O-I nanohybrids (on glass) decreased from 13 to $1.5 \mathrm{~nm}$ after the pressing process. Although a study has been performed to reduce the surface roughness of graphene oxide (rGO) using a polyethersulfone film [51], it was challenging to fabricate a graphene film with high surface smoothness on a rigid substrate, owing to the brittleness of the rigid glass. 

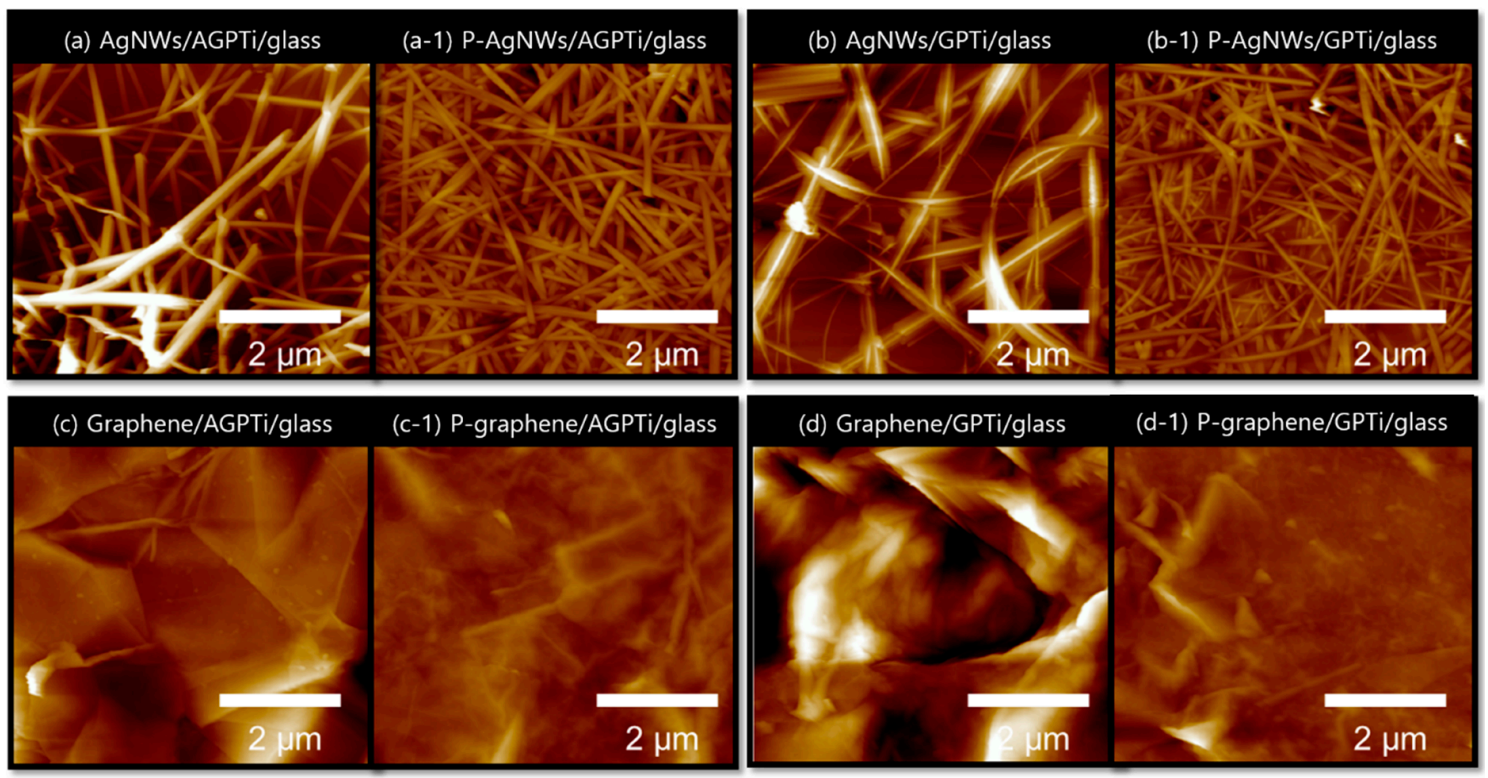

Figure 6. AFM images of the AgNWs and graphene on AGPTi and GPTi before ((a) AgNWs/AGPTi/glass, (b) AgNWs/GPTi/glass, (c) graphene/AGPTi/glass, and (d) graphene/GPTi/glass), and after ((a-1) P-AgNWs/AGPTi/glass, (b-1) P-AgNWs/GPTi/glass, (c-1) P-graphene/AGPTi/glass, and (d-1) P-graphene/GPTi/glass) the mechanical pressing process.

The AGPTi and GPTi O-I nanohybrid layers were further investigated to determine their suitability for roll-to-roll-based printed electronics using gravure printing. Gravure printing is a promising roll-to-roll-based process providing a high-speed deposition of functional materials in a large area with low cost, thus enabling very high productivity [52]. Fundamentally, this method involves three steps: filling of the pattern with ink, removal of excess ink from the surface, and transfer of the ink to the substrate. Therefore, an investigation of the relationship between the ink and substrate is necessary to improve the pattern fidelity. Using the optimized AgNWs and graphene ink for the gravure printing process, conductive lines of AgNWs (Figure 7a for AGPTi and Figure $7 \mathrm{~b}$ for GPTi) and graphene (Figure 7c for AGPTi and Figure 7d for GPTi) were fabricated at a printing speed of 3 $\mathrm{cm} / \mathrm{s}$. The continuous line morphologies demonstrate the proper wettability and printability of the AGPTi and GPTi O-I nanohybrid layers to the desired materials for patterning. Trenches of $500 \mu \mathrm{m}$ yielded lines of gravure-printed AgNWs/AGPTi/PET with widths of $490 \pm 15 \mu \mathrm{m}$ and gravure-printed graphene/AGPTi/PET with widths of $470 \pm 12 \mu \mathrm{m}$. However, narrower lines were obtained on the GPTi O-I nanohybrid layer, with widths of $481 \pm 15 \mu \mathrm{m}$ (gravure-printed AgNWs/GPTi/PET) and $424 \pm 15 \mu \mathrm{m}$ (gravure-printed graphene/GPTi/PET), as shown in Figure 8a. This can be explained in terms of surface energy affecting the spreading of the water-based solution. As the contact angle of DI water on the GPTi O-I nanohybrid was $91.3^{\circ}$, which is $12^{\circ}$ higher than that for the AGPTi nanohybrid, a lower surface energy induced by the lower polar energy was expected, leading to the reduced line width. In order to reveal the electrical properties of the printed AgNW and graphene lines, conductivity measurements were performed. The results of line resistance measurements are shown in Figure $8 \mathrm{~b}$. All of the printed lines (500-325 $\mu \mathrm{m}$ ) were electrically continuous over the entire line lengths of $2 \mathrm{~cm}$ with a standard deviation lower than $10 \%$, indicating their high continuity and uniformity. In addition, the tendency of decrease in line resistance as a function of the line width is attributed to the increase in electrical area for electron transport. Decreases in the line resistances, from 32 to $1 \mathrm{k} \Omega / \mathrm{mm}$ for the gravure-printed AgNWs/GPTi/PET and from 25 to $0.4 \mathrm{k} \Omega / \mathrm{mm}$ for the gravure-printed graphene/AGPTi/PET, were observed. 

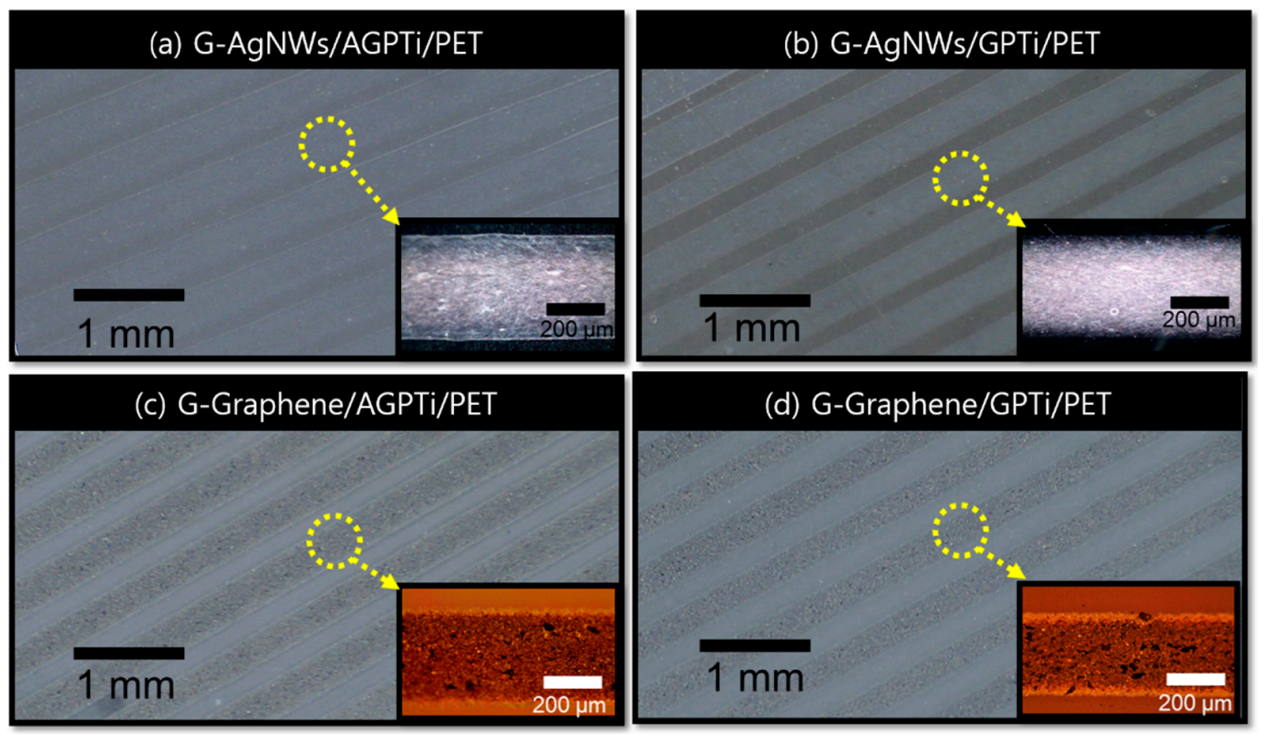

Figure 7. Gravure-printed lines of AgNWs and graphene on AGPTi ((a) G-AgNWs/AGPTi/PET and (c) G-graphene/AGPTi/PET) and GPTi ((b) G-AgNWs/GPTi/PET and (d) G-graphene/GPTi/PET), which were predeposited on PET.
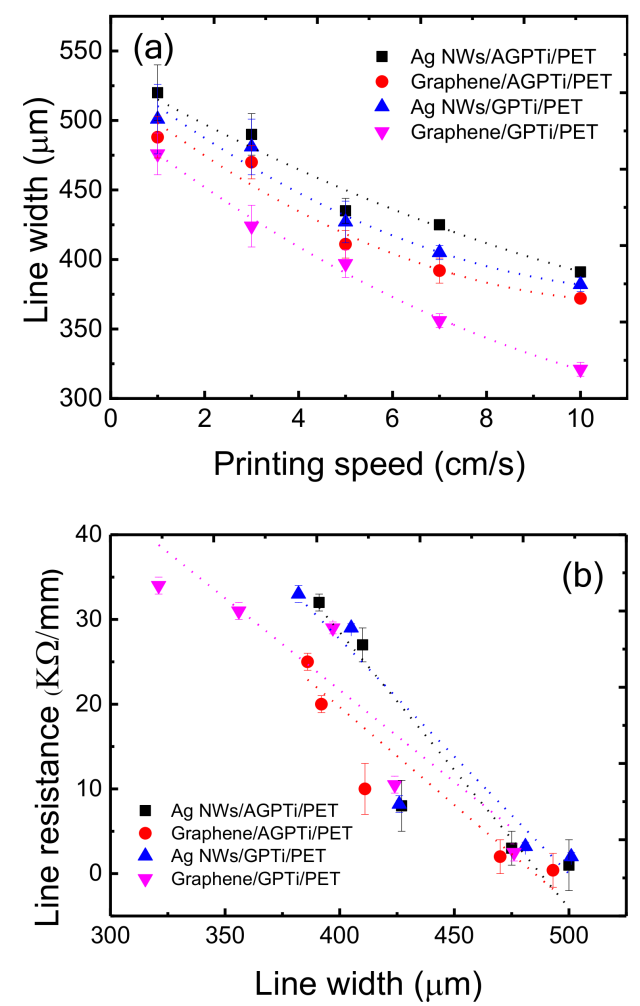

Figure 8. (a) Relationship between the printing speed and line width. (b) Change in line resistance as a function of the line width.

The high flexibilities of the films with the AGPTi and GPTi O-I nanohybrids were demonstrated by characterizing mechanical properties upon measurements of the line resistances over a large number of bending cycles. Figure 9a shows the normalized line resistances as a function of the bending cycle; no measurable loss of line resistance over the entire number-of-cycles range was observed for the AGPTi O-I nanohybrid (Figure $9 \mathrm{~b}, \mathrm{c}$ ) at a radius of curvature of $5 \mathrm{~mm}$. By contrast, a significant decrease in line resistance was observed for the GPTi O-I nanohybrid during the 1000 bending cycles. 
In particular, the gravure-printed AgNWs/GPTi/PET (Figure 9d) had a normalized resistance over 10 at 100 bending cycles, which was ten times higher than that of the gravure-printed graphene/GPTi/PET (Figure 9e). This is attributed to the easier breaking of AgNWs under the bending pressure. The origin of the higher endurance of the AGPTi O-I nanohybrid against the bending pressure (Figure 9d,e), than that of the GPTi nanohybrid, is attributed to the chemical structure of AFAP in the AGPTi O-I nanohybrid. As mentioned above, AFAP consists of two types of polymer chains: polyethylene oxide-based hydrophilic and polypropylene oxide-based hydrophobic. The amphiphilic AFAP could colloidally stabilize the O-I nanohybrid sols during the hydrolytic condensation polymerization. In the thin-film state, the polymer segment of AFAP can provide the flexibility between O-I nanohybrid particles forming the film, which contributed to the endurance of the AGPTi O-I nanohybrid against the bending pressure. Therefore, AGPTi has desirable mechanical properties enabling the fabrication of highly functional flexible devices [53].
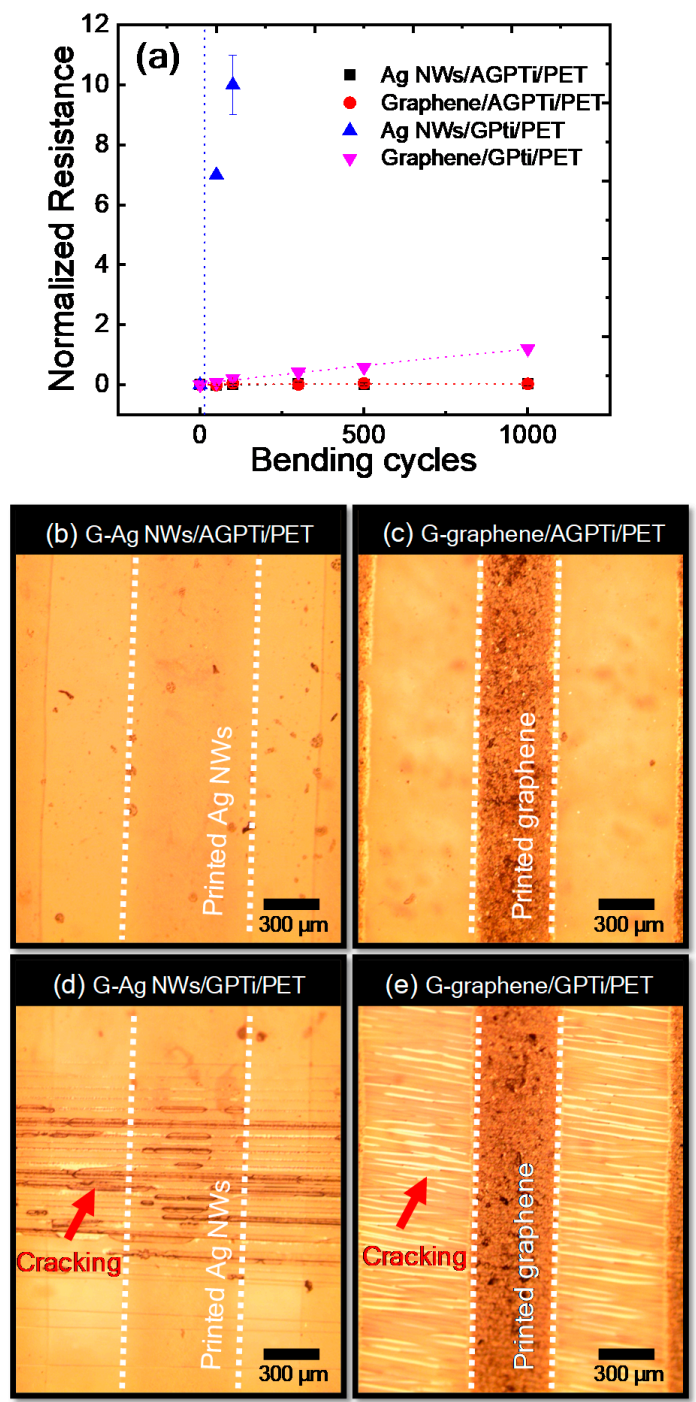

Figure 9. (a) Normalized line resistances of the gravure-printed AgNWs and graphene on AGPTi and GPTi as a function of the bending cycle. Optical images of the gravure-printed AgNWs and graphene on AGPTi ((b) G-AgNWs/AGPTi/PET and (c) G-graphene/AGPTi/PET) and GPTi ((d) G-AgNWs/GPTi/PET and (e) G-graphene/GPTi/PET) after 1000 bending cycles.

\section{Conclusions}

AGPTi, an O-I nanohybrid obtained by incorporating the AFAP precursor into the $\mathrm{SiO}_{2}-\mathrm{TiO}_{2}$ hybrid network, was successfully utilized as a buffer layer to fabricate a flexible, transparent, and stable 
conductive substrate based on solution-processed AgNWs and graphene under ambient conditions. The transmittance of the AgNWs spin-coated on AGPTi/glass was $99.4 \%$ at $550 \mathrm{~nm}$. The high adhesion strength was demonstrated by the 3M tape test; no visible changes in the AgNWs were observed. In addition, AGPTi had an important role in the reductions in the sheet resistances of the pressed AgNWs and graphene on AGPTi, from 5 to $1.5 \Omega$ /sq and from 700 to $600 \Omega /$ sq, respectively. This was mainly attributed to the enhanced connection of wire-to-wire junctions of the AgNWs, and enlargement in the contact area between graphene particles during the pressing process. Furthermore, AGPTi acted as a highly functional buffer layer, absorbing the applied pressure between the conductive materials and rigid substrate, where the AgNWs and graphene particles provided stable structural networks. In order to investigate the applicability of the AGPTi layer in printed electronics, gravure printings of the optimized AgNWs and graphene ink were performed on the flexible substrates at $3 \mathrm{~cm} / \mathrm{s}$, yielding line widths of $490 \pm 15 \mu \mathrm{m}$ (gravure-printed AgNWs/AGPTi/PET) and $470 \pm 12 \mu \mathrm{m}$ (gravure-printed graphene/AGPTi/PET), for $500 \mu \mathrm{m}$ gravure patterns. The good mechanical properties of AGPTi were demonstrated by a bending test (1000 cycles), which showed the high flexibility of the structure with the AGPTi O-I nanohybrid. Therefore, AGPTi as a buffer layer for printed electronics can increase the application of conductive materials to high-end electronic devices requiring high transparencies, flexibilities, and adhesion properties.

Author Contributions: Conceptualization, S.H.K.; Data curation, X.L.; Funding acquisition, T.K.A. and S.L.; Investigation, N.K.; Writing—original draft, J.K., S.L. and S.H.K.; Writing—review \& editing, S.Y. and T.K.A.

Acknowledgments: This work was supported by the National Research Foundation of Korea (NRF) grant funded by the Korea government (NRF-2018R1D1A1D07043938), and also by “Human Resources Program in Energy Technology" of the Korea Institute of Energy Technology Evaluation and Planning (KETEP), granted financial resource from the Ministry of Trade, Industry \& Energy, Korea (No. 20174030201760). This research was also supported by a National Research Foundation of Korea (NRF) grant funded by the Korean government (MSIP) (2017R1C1B2002888).

Conflicts of Interest: The authors declare no conflict of interest.

\section{References}

1. Gelinck, G.H.; Huitema, H.E.A.; Van Veenendaal, E.; Cantatore, E.; Schrijnemakers, L.; Van der Putten, J.B.P.H.; Geuns, T.C.T.; Beenhakkers, M.; Giesbers, J.B.; Huisman, B.H.; et al. Flexible Active-Matrix Displays and Shift Registers Based on Solution-Processed Organic Transistors. Nat. Mater. 2004, 3, 106-110. [CrossRef] [PubMed]

2. Pang, C.; Lee, C.; Suh, K.Y. Recent Advances in Flexible Sensors for Wearable and Implantable Devices. J. Appl. Polym. Sci. 2013, 130, 1429-1441. [CrossRef]

3. Bauer, S.; Bauer-Gogonea, S.; Graz, I.; Kaltenbrunner, M.; Keplinger, C.; Schwodiauer, R. 25th Anniversary Article: A Soft Future: From Robots and Sensor Skin to Energy Harvesters. Adv. Mater. 2014, 26, 149-162. [CrossRef]

4. Beeby, S.P.; Torah, R.N.; Tudor, M.J.; Glynne-Jones, P.; O’Donnell, T.; Saha, C.R.; Roy, S. A Micro Electromagnetic Generator for Vibration Energy Harvesting. J. Micromech. Microeng. 2007, 17, 1257-1265. [CrossRef]

5. Lee, S.H.; Jeong, C.K.; Hwang, G.T.; Lee, K.J. Self-Powered Flexible Inorganic Electronic System. Nano Energy 2015, 14, 111-125. [CrossRef]

6. Wang, Z.L.; Wu, W.Z. Nanotechnology-Enabled Energy Harvesting for Self-Powered Micro-/Nanosystems. Angew. Chem. Int. Edit. 2012, 51, 11700-11721. [CrossRef]

7. Qi, Y.; Kim, J.; Nguyen, T.D.; Lisko, B.; Purohit, P.K.; McAlpine, M.C. Enhanced Piezoelectricity and Stretchability in Energy Harvesting Devices Fabricated from Buckled PZT Ribbons. Nano Lett. 2011, 11, 1331-1336. [CrossRef]

8. Tarascon, J.M.; Armand, M. Issues and Challenges Facing Rechargeable Lithium Batteries. Nature 2001, 414, 359-367. [CrossRef] 
9. Lorincz, K.; Malan, D.J.; Fulford-Jones, T.R.F.; Nawoj, A.; Clavel, A.; Shnayder, V.; Mainland, G.; Welsh, M.; Moulton, S. Sensor Networks for Emergency Response: Challenges and Opportunities. IEEE Pervas. Comput. 2004, 3, 16-23. [CrossRef]

10. Tate, J.; Rogers, J.A.; Jones, C.D.W.; Vyas, B.; Murphy, D.W.; Li, W.J.; Bao, Z.A.; Slusher, R.E.; Dodabalapur, A.; Katz, H.E. Anodization and Microcontact Printing on Electroless Silver:Solution-Based Fabrication Procedures for Low-Voltage Electronic Systems with Organic Active Components. Langmuir 2000, 16, 6054-6060. [CrossRef]

11. Li, Y.N.; Wu, Y.L.; Ong, B.S. Facile Synthesis of Silver Nanoparticles Useful for Fabrication of High-Conductivity Elements for Printed Electronics. J. Am. Chem. Soc. 2005, 127, 3266-3267. [CrossRef] [PubMed]

12. Secor, E.B.; Prabhumirashi, P.L.; Puntambekar, K.; Geier, M.L.; Hersam, M.C. Inkjet Printing of High Conductivity, Flexible Graphene Patterns. J. Phys. Chem. Lett. 2013, 4, 1347-1351. [CrossRef]

13. Tekin, E.; Smith, P.J.; Schubert, U.S. Inkjet Printing as a Deposition and Patterning Tool for Polymers and Inorganic Particles. Soft Matter 2008, 4, 703-713. [CrossRef]

14. Li, J.; Zhao, Y.; Tan, H.S.; Guo, Y.L.; Di, C.A.; Yu, G.; Liu, Y.Q.; Lin, M.; Lim, S.H.; Zhou, Y.H.; et al. A Stable Solution-Processed Polymer Semiconductor with Record High-Mobility for Printed Transistors. Sci. Rep. 2012, 2, 754. [CrossRef]

15. Lau, P.H.; Takei, K.; Wang, C.; Ju, Y.; Kim, J.; Yu, Z.B.; Takahashi, T.; Cho, G.; Javey, A. Fully Printed, High Performance Carbon Nanotube Thin-Film Transistors on Flexible Substrates. Nano Lett. 2013, 13, 3864-3869. [CrossRef] [PubMed]

16. Rim, Y.S.; Bae, S.H.; Chen, H.J.; De Marco, N.; Yang, Y. Recent Progress in Materials and Devices Toward Printable and Flexible Sensors. Adv. Mater. 2016, 28, 4415-4440. [CrossRef]

17. Huang, D.; Liao, F.; Molesa, S.; Redinger, D.; Subramanian, V. Plastic-Compatible Low Resistance Printable Gold Nanoparticle Conductors for Flexible Electronics. J. Electrochem. Soc. 2003, 150, G412-G417. [CrossRef]

18. Logothetidis, S. Flexible Organic Electronic Devices: Materials, Process and Applications. Mater. Sci. Eng. $B-A d v$. 2008, 152, 96-104. [CrossRef]

19. Zarek, M.; Layani, M.; Cooperstein, I.; Sachyani, E.; Cohn, D.; Magdassi, S. 3D Printing of Shape Memory Polymers for Flexible Electronic Devices. Adv. Mater. 2016, 28, 4449-4454. [CrossRef] [PubMed]

20. Blanchet, G.B.; Loo, Y.L.; Rogers, J.A.; Gao, F.; Fincher, C.R. Large Area, High Resolution, Dry Printing of Conducting Polymers for Organic Electronics. Appl. Phys. Lett. 2003, 82, 463-465. [CrossRef]

21. Na, S.I.; Kim, S.S.; Jo, J.; Kim, D.Y. Efficient and Flexible ITO-Free Organic Solar Cells Using Highly Conductive Polymer Anodes. Adv. Mater. 2008, 20, 4061-4067. [CrossRef]

22. Ito, S.; Ha, N.L.C.; Rothenberger, G.; Liska, P.; Comte, P.; Zakeeruddin, S.M.; Pechy, P.; Nazeeruddin, M.K.; Gratzel, M. High-Efficiency (7.2\%) Flexible Dye-Sensitized Solar Cells with Ti-Metal Substrate for Nanocrystalline-TiO 2 Photoanode. Chem. Commun. 2006, 38, 4004-4006. [CrossRef] [PubMed]

23. Park, Y.S.; Choi, K.H.; Kim, H.K. Room Temperature Flexible and Transparent ITO/Ag/ITO Electrode Grown on Flexile PES Substrate by Continuous Roll-To-Roll Sputtering for Flexible Organic Photovoltaics. J. Phys. D. Appl. Phys. 2009, 42, 235109. [CrossRef]

24. Lin, Y.C.; Li, J.Y.; Yen, W.T. Low Temperature ITO Thin Film Deposition on PES Substrate Using Pulse Magnetron Sputtering. Appl. Surf. Sci. 2008, 254, 3262-3268. [CrossRef]

25. Huang, L.M.; Chen, C.H.; Wen, T.C. Development and Characterization of Flexible Electrochromic Devices Based on Polyaniline and Poly(3,4-Ethylenedioxythiophene)-Poly(Styrene Sulfonic Acid). Electrochim. Acta 2006, 51, 5858-5863. [CrossRef]

26. Hwang, B.; Li, X.; Kim, S.H.; Lim, S. Effect of Carbon Nanotube Addition on Mechanical Reliability of Ag Nanowire Network. Mater. Lett. 2017, 198, 202-205. [CrossRef]

27. Li, X.; Lee, G.S.; Park, S.H.; Kong, H.; An, T.K.; Kim, S.H. Direct Writing of Silver Nanowire Electrodes Via Dragging Mode Electrohydrodynamic Jet Printing for Organic Thin Film Transistors. Org. Electron. 2018, 62, 357-365. [CrossRef]

28. Lee, H.-J.; Song, Y.-S.; An, T.K.; Choi, W.-K.; Kim, S.-R. Ultrasmooth transparent conductive hybrid films of reduced graphene oxide and single-walled carbon nanotube by ultrasonic spraying. Synth. Met. 2016, 221, 340-344. [CrossRef] 
29. Dang, Y.; Holmes, D.E.; Zhao, Z.Q.; Woodard, T.L.; Zhang, Y.B.; Sun, D.Z.; Wang, L.Y.; Nevin, K.P.; Lovley, D.R. Enhancing Anaerobic Digestion of Complex Organic Waste with Carbon-Based Conductive Materials. Bioresour. Technol. 2016, 220, 516-522.

30. Deng, B.; Hsu, P.C.; Chen, G.C.; Chandrashekar, B.N.; Liao, L.; Ayitimuda, Z.; Wu, J.X.; Guo, Y.F.; Lin, L.; Zhou, Y.; et al. Roll-to-Roll Encapsulation of Metal Nanowires Between Graphene and Plastic Substrate for High-Performance Flexible Transparent Electrodes. Nano Lett. 2015, 15, 4206-4213. [CrossRef] [PubMed]

31. Li, X.L.; Kim, K.W.; Joo, S.W.; Seo, J.; Lee, J.; An, T.K.; Kim, S.H. Facile Method for Enhancing Conductivity of Printed Carbon Nanotubes Electrode via Simple Rinsing Process. Org. Electron. 2017, 47, 174-180. [CrossRef]

32. Guarnera, S.; Abate, A.; Zhang, W.; Foster, J.M.; Richardson, G.; Petrozza, A.; Snaith, H.J. Improving the Long-Term Stability of Perovskite Solar Cells with a Porous $\mathrm{Al}_{2} \mathrm{O}_{3}$ Buffer Layer. J. Phys. Chem. Lett. 2015, 6, 432-437. [CrossRef] [PubMed]

33. Ren, Z.W.; Wang, J.; Pan, Z.X.; Zhao, K.; Zhang, H.; Li, Y.; Zhao, Y.X.; Mora-Sero, I.; Bisquert, J.; Zhong, X.H. Amorphous $\mathrm{TiO}_{2}$ Buffer Layer Boosts Efficiency of Quantum Dot Sensitized Solar Cells to over $9 \%$. Chem. Mater. 2015, 27, 8398-8405. [CrossRef]

34. Kavan, L.; Steier, L.; Gratzel, M. Ultrathin Buffer Layers of $\mathrm{SnO}_{2}$ by Atomic Layer Deposition: Perfect Blocking Function and Thermal Stability. J. Phys. Chem. C 2017, 121, 342-350. [CrossRef]

35. Zhan, C.X.; Yu, G.Q.; Lu, Y.; Wang, L.Y.; Wujcik, E.; Wei, S.Y. Conductive Polymer Nanocomposites: A Critical Review of Modern Advanced Devices. J. Mater. Chem. C 2017, 5, 1569-1585. [CrossRef]

36. Manthiram, A.; Li, L.J. Hybrid and Aqueous Lithium-Air Batteries. Adv. Energy. Mater. 2015, 5, 1401302. [CrossRef]

37. Ababtain, K.; Babu, G.; Susarla, S.; Gullapalli, H.; Masurkar, N.; Ajayan, P.M.; Arava, L.M.R. Porous Graphene Current Collectors Filled with Silicon as High-Performance Lithium Battery Anode. Mater. Res. Express 2018, 5, 014004. [CrossRef]

38. Kim, N.; Kim, S.H.; Kim, J. Colloidally Stable Organic- Inorganic Hybrid Nanoparticles Prepared Using Alkoxysilane-Functionalized Amphiphilic Polymer Precursors and Mechanical Properties of Their Cured Coating Film. J. Ind. Eng. Chem. 2018, 68, 209-219. [CrossRef]

39. Kim, J.; Wainaina, J.; Na, J.S. Synthesis of Amphiphilic Silica/Polymer Composite Nanoparticles as Water-Dispersible Nano-Absorbent for Hydrophobic Pollutants. J. Ind. Eng. Chem. 2011, 17, 681-690. [CrossRef]

40. Sun, Y.G.; Yin, Y.D.; Mayers, B.T.; Herricks, T.; Xia, Y.N. Uniform Silver Nanowires Synthesis by Reducing $\mathrm{AgNO}_{3}$ with Ethylene Glycol in the Presence of Seeds and Poly(Vinyl Pyrrolidone). Chem. Mater. 2002, 14, 4736-4745. [CrossRef]

41. Kim, Y.H.; Cho, H.; Heo, J.H.; Kim, T.S.; Myoung, N.; Lee, C.L.; Im, S.H.; Lee, T.W. Multicolored Organic/Inorganic Hybrid Perovskite Light-Emitting Diodes. Adv. Mater. 2015, 27, 1248-1254. [CrossRef] [PubMed]

42. Yu, S.; Guo, Z.G.; Liu, W.M. Biomimetic Transparent and Superhydrophobic Coatings: From Nature and Beyond Nature. Chem. Commun. 2015, 51, 1775-1794. [CrossRef] [PubMed]

43. Malgras, V.; Ji, Q.M.; Kamachi, Y.; Mori, T.; Shieh, F.K.; Wu, K.C.W.; Ariga, K.; Yamauchi, Y. Templated Synthesis for Nanoarchitectured Porous Materials. B. Chem. Soc. Jpn. 2015, 88, 1171-1200. [CrossRef]

44. Geng, H.Z.; Kim, K.K.; So, K.P.; Lee, Y.S.; Chang, Y.; Lee, Y.H. Effect of Acid Treatment on Carbon Nanotube-Based Flexible Transparent Conducting Films. J. Am. Chem. Soc. 2007, 129, 7758. [CrossRef]

45. Pham, V.H.; Cuong, T.V.; Hur, S.H.; Shin, E.W.; Kim, J.S.; Chung, J.S.; Kim, E.J. Fast and Simple Fabrication of a Large Transparent Chemically-Converted Graphene Film by Spray-Coating. Carbon 2010, 48, 1945-1951. [CrossRef]

46. Madaria, A.R.; Kumar, A.; Ishikawa, F.N.; Zhou, C.W. Uniform, Highly Conductive, and Patterned Transparent Films of a Percolating Silver Nanowire Network on Rigid and Flexible Substrates using a Dry Transfer Technique. Nano Res. 2010, 3, 564-573. [CrossRef]

47. Gaskell, P.E.; Skulason, H.S.; Rodenchuk, C.; Szkopek, T. Counting Graphene Layers on Glass via Optical Reflection Microscopy. Appl. Phys. Lett. 2009, 94, 143101. [CrossRef]

48. Lee, S.H.; Lim, S.; Kim, H. Smooth-Surface Silver Nanowire Electrode with High Conductivity and Transparency on Functional Layer Coated Flexible Film. Thin Solid Films 2015, 589, 403-407. [CrossRef] 
49. Marinho, B.; Ghislandi, M.; Tkalya, E.; Koning, C.E.; de With, G. Electrical Conductivity of Compacts of Graphene, Multi-Wall Carbon Nanotubes, Carbon Black, and Graphite Powder. Powder Technol. 2012, 221, 351-358. [CrossRef]

50. Gaynor, W.; Burkhard, G.F.; McGehee, M.D.; Peumans, P. Smooth Nanowire/Polymer Composite Transparent Electrodes. Adv. Mater. 2011, 23, 2905-2910. [CrossRef]

51. Shin, K.H.; Jang, Y.; Kim, B.S.; Jang, J.; Kim, S.H. Highly Conductive Reduced Graphene Oxide Produced via Pressure-Assisted Reduction at Mild Temperature for Flexible and Transparent Electrodes. Chem. Commun. 2013, 49, 4887-4889. [CrossRef] [PubMed]

52. Secor, E.B.; Lim, S.; Zhang, C.D.; Frisbie, H.; Francis, L.F.; Hersam, M.C. Gravure Printing of Graphene for Large-area Flexible Electronics. Adv. Mater. 2014, 26, 4533-4538. [CrossRef] [PubMed]

53. Gunes, F.; Shin, H.J.; Biswas, C.; Han, G.H.; Kim, E.S.; Chae, S.J.; Choi, J.Y.; Lee, Y.H. Layer-by-Layer Doping of Few-Layer Graphene Film. ACS Nano 2010, 4, 4595-4600. [CrossRef] [PubMed]

2019 by the authors. Licensee MDPI, Basel, Switzerland. This article is an open access article distributed under the terms and conditions of the Creative Commons Attribution (CC BY) license (http://creativecommons.org/licenses/by/4.0/). 